\title{
Giant pituitary adenoma associated with extra diaphragmatic extension as multiple nubbing into subarachnoid space: Uncommon entity
}

\author{
Guru Dutta Satyarthee* and Satish Verma \\ Department of Neurosurgery, Neurosciences Centre, AIIMS, New Delhi, India
}

\begin{abstract}
Surgical management of giant pituitary adenomas possess challenges due to presence of optic nerves, internal carotid arteries and hypothalamus in and around seller and para seller region. The extent of tumor resection governs the efficacy of surgical treatment. However surgical extent of resection depends on para seller extension, multiple compartmental extension, and relation with displaced and distorted neurovascular structures. However, phenomenon of extra diaphragmatic extension of giant pituitary adenoma as multiple nubbing of pituitary adenoma into subarachnoid space is extremely uncommon. To the best knowledge of authors current case represents first case in the western literature. In such situation, attempting microscopic transsphenoidal surgical approach may prove much hazardous but endoscopic surgery can offer best option. Authors discuss importance of extra diaphragmatic extension and possible role of various surgical modality and endoscopic surgery and pertinent literature is reviewed briefly as awareness of such pathology is very important for every emergency care physician, critical care team including neurosurgeons and, radiologist and allied team to make a sound management strategy.
\end{abstract}

\section{Introduction}

Pituitary adenomas account for about 10 to $15 \%$ of all intracranial tumors and characteristically presents with endocrinological dysfunctions including either hypopituitarism or functional hormonal secreting [1,2]. Along with visual disturbances and mass effect on surrounding neurovascular structure [3]. This giant pituitary adenoma usually requires multimodality treatment, but usual first modality of definitive treatment remains surgical extirpation, followed by radiosurgery for residual pituitary adenoma or repeat surgical intervention. The usual surgical approaches for these lesions are either the transsphenoidal or transcranial approach [2-4].

Extra diaphragmatic extension of giant pituitary adenoma as multiple nubbing of pituitary adenoma into subarachnoid space is extremely uncommon. The multiple nubbing of suprasellar extension is caused due to incompetency of diaphragm selle around the pituitary stalk, through which, adenoma extending into subarachnoid plane and further causes encasement of optic nerve, chiasma and vessels. In such situation, attempting transsphenoidal approach may prove much hazardous. Awareness of such pathology is very important for every neurosurgeons and allied team [3-5].

\section{Case illustration}

A-58-year non-diabetic, non-hypertensive male presented with history of three episodes of left sided focal motor seizure with secondary generalization over the last three months. On examination, vitals were stable with no phenotypic marker of endocrinopathy were present. Neurological examination revealed visual acuity of $6 / 18$ on both sides with bitemporal hemianopia. Routine hemogram, biochemistry and hormonal levels were within normal limits. MRI brain revealed presence of a giant pituitary adenoma with presence of multiple nubbins, which were extending into extra diaphragmatic subarachnoid space (Figures 1 and 2). He underwent endoscopic transsphenoidal decompression of pituitary adenoma. During surgery direct visualization under endoscopic assistance to excision of giant pituitary adenoma along with extra diaphragmatic extension into subarachnoid spaces led to complete excision. In the post-operative period, had episodes of diabetes insipidus managed with vasopressin nasal spray. He was discharged from hospital on tenth post-operative day.

\section{Discussion}

Pituitary adenomas with a diameter larger than $4 \mathrm{~cm}$ in sizes, or multicompartmental or extending up to floor of third ventricle is generally assigned as giant pituitary adenomas. It accounts for about $5 \%$ of the pituitary adenomas. Giant pituitary adenoma can present presents with progressive visual deterioration, endocrinological dysfunctions, focal neurological deficit or features of raised intracranial pressure. Detailed hormonal workup along with assessment of comorbidity is essential prior to planning of surgical management [1-3]. Magnetic resonance imaging is considered as gold standard method for diagnoses, as it delineates, epicenter, extent, parasellar involvement, sub-frontal or extension into middle cranial fossa and relation of carotid vessels and cavernous sinus and associated obstructive hydrocephalus

*Correspondence to: Guru Dutta Satyarthee, Department of Neurosurgery, Neurosciences Centre, AIIMS, New Delhi, India, Tel: 0112658 8500; E-mail: drguruduttaaiims@gmail.com

Key words: giant pituitary adenoma, extra-diaphragmatic extension, MRI, surgical risk, awareness

Received: August 10, 2018; Accepted: August 24, 2018; Published: August 28, 2018 


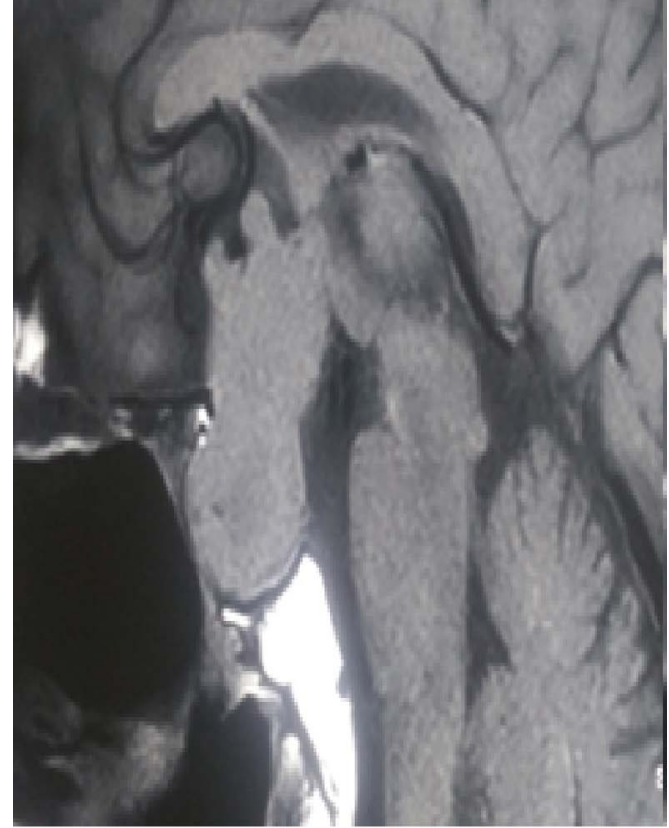

Figure 1. Magnetic resonance imaging, sagittal section, T1 weighted images showing giant pituitary adenoma with presence of multiple nubbins on superior surface representing extra diaphragmatic subarachnoid extension

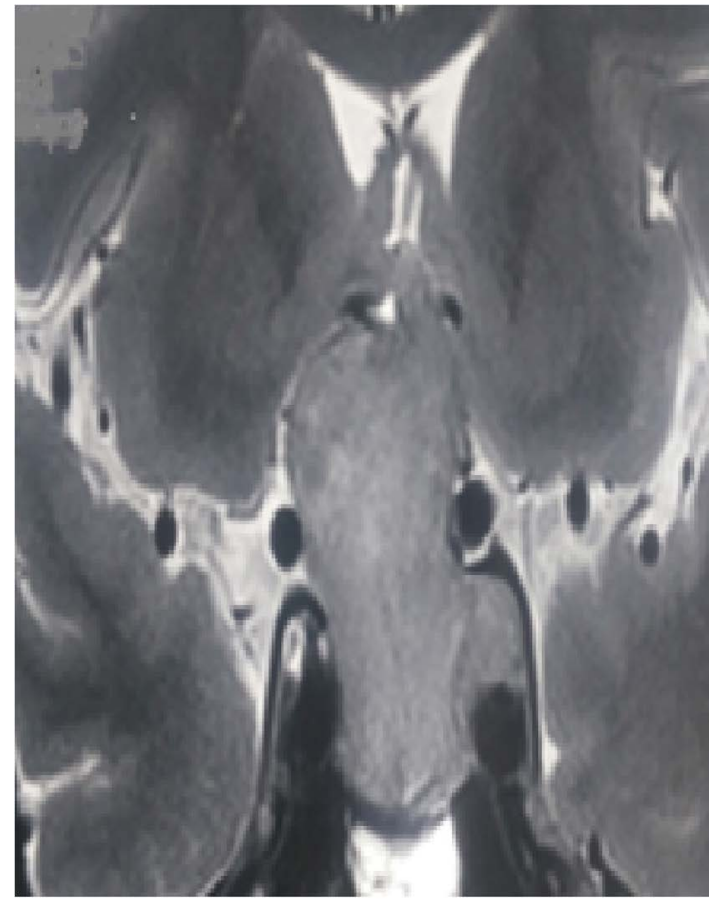

Figure 2. Cranial Magnetic resonance imaging, coronal section, $\mathrm{T} 2$ weighted images showing multiple subarachnoid nubbins with extra diaphragmatic subarachnoid extension in a ca se of giant pituitary adenoma

and any previous or present episode of pituitary apoplexy. MRI also detects presence of extra diaphragmatic extension into subarachnoid space as multiple nubbing of pituitary adenoma. The multiple nubbing of suprasellar extension is caused due to incompetency of diaphragm sella around the pituitary stalk, through which, adenoma extending into subarachnoid plane and further causes encasement of optic nerve, chiasma and carotid anterior cerebral arteries. In such situation, attempting transsphenoidal approach may prove much hazardous.
Awareness of such pathology is very important for proper management $[2,3]$. These giant pituitary adenomas usually require multimodality treatment, but usual first modality of definitive treatment remains surgical extirpation. The rate of gross and near-total tumor removal after transsphenoidal, transcranial, or combined procedures ranges from $14.7 \%$ to $74 \%$ in cases of giant pituitary adenomas, according to previously published reports [4-6]. The transsphenoidal approach is considered as preferred surgical modality for the surgical excision of most of the pituitary adenomas, as compared with transcranial routes [5]. Usually endoscopic transsphenoidal surgery represents the gold standard treatment for even giant pituitary adenoma in experienced hand, however, such cases with extra diaphragmatic subarachnoid extension are more suitable for transcranial surgery as surgical decompression is carried out under vision with relatively better chances of preservation of optic nerves, chiasma, and vessel, although transcranial approach carry its own disadvantages. The multiple nubbing of suprasellar extension of pituitary adenoma in our patient is caused due to the diaphragm sella becoming compromised around the pituitary stalk and adenoma extending into subarachnoid plane and encasing around optic chiasma and internal carotid artery and anterior cerebral artery and other branches, making transsphenoidal approach much more hazardous [6]. The role of radiation therapy or radiotherapies in preventing the late recurrence or control the residual tumor which was impossible to remove by surgery especially in the cavernous sinus invasion is important [5].

\section{Conclusion}

Majority of giant pituitary adenoma can be managed with proper derailed hormonal assay, visual acuity and field charting and neuroimaging including magnetic resonance imaging study. However, every health care tram should be aware of possibility of presence of extra diaphragmatic extension into subarachnoid space as multiple nubbing of pituitary adenoma. The suprasellar extension into subarachnoid space as nubbins is caused by incompetency of diaphragm sella, which typically closely cover around the pituitary stalk, and causes encasement of optic nerve, chiasma and carotid anterior cerebral arteries.

\section{References}

1. Goel A, Nadkarni T, Muzumdar D, Desai K, Phalke U, et al. (2004) Giant pituitary tumors: a study based on surgical treatment of 118 cases. Surg Neurol 61: 436-445. [Crossref]

2. Vakharia RM, Kremen R, Vakharia A, Adarkwah O, Anderson G (2016) Anterior and posterior suprasellar extensions of a symmetrical trilobed nonfunctional giant pituitary adenoma in the sagittal plane: a case report and review of literature. Radiol Case Rep 11: 419-424. [Crossref]

3. Zhang X, Fei Z, Zhang Z, Liu W, Chen Y (1999) Management of non- functioning pituitary adenomas with suprasellar extensions by transsphenoidal microsurgery. Surg Neurol 52: 380-385. [Crossref]

4. Satyarthee GD, Mahapatra AK (2002) Seizure as presentation of pituitary adenomas; series of four cases. Neurosciences Today 6: 167-169.

5. Mortini P, Barzaghi R, Lossa M, Boari N, Giovanelli M (2007) Surgical treatment of giant pituitary adenomas: Strategies and results in a series of 95 consecutive patients. Neurosurgery. 60: 993-1004. [Crossref]

6. Sinha S, Sharma BS (2010) Giant pituitary adenomas-an enigma revisited. Microsurgical treatment strategies and outcome in a series of 250 patients. $B r J$ Neurosurg 24: 31-39. [Crossref]

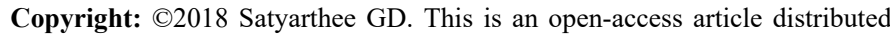
under the terms of the Creative Commons Attribution License, which permits unrestricted use, distribution, and reproduction in any medium, provided the original author and source are credited. 